Elsevier required licence: (C) $<2020>$. This manuscript version is made available under the CC-BY-NCND 4.0 license http://creativecommons.org/licenses/by-nc-nd/4.0/

The definitive publisher version is available online at

[https://www.sciencedirect.com/science/article/abs/pii/S0957417420305686?via\%3Dihub] 


\title{
Rotation Invariant Angle-Density based Features for an Ice Image Classification System
}

\author{
${ }^{1}$ Shengkai Yue, ${ }^{2}$ Palaiahnakote Shivakumara, ${ }^{1}$ Minglei Yuan, ${ }^{1}$ Tong Lu, ${ }^{3}$ Michael Blumenstein, \\ ${ }^{4}$ Hualu Zhang and ${ }^{5} \mathrm{G}$. Hemantha Kumar \\ ${ }^{1}$ National Key Lab for Novel Software Technology, Nanjing University, China. Email: \\ shengkai@smail.nju.edu.cn, lutong@nju.edu.cn,mlyuan@smail.nju.edu.cn \\ ${ }^{2}$ Faculty of Computer Science and Information Technology, University of Malaya, Malaysia. \\ Email: shiva@um.edu.my \\ ${ }^{3}$ University of Technology Sydney (UTS), Sydney, Australia. michael.blumenstein@uts.edu.au \\ ${ }^{4}$ Information Technology \& Communication Company Nari Group Corporation, Nanjing, China \\ zhanghualu@sgepri.sgcc.com.cn \\ ${ }^{5}$ Department of Studies in Computer Science, University of Mysore, India. Email: \\ ghk.2007@yahoo.com.
}

\begin{abstract}
One of the natural disasters which cause economic loss and are a serious threat to society are ice covering phenomena for overhead transmission. This paper presents a new method for ice and non-ice image classification to improve ice detection results. The proposed method explores wavelet decomposition to extract robust features, such as those invariant to rotation, scaling and thickness of ice for classification. The proposed method estimates the average of the high frequency sub-bands for each level. Then it obtains Canny edge components for the average wavelet image at each level. The proposed method studies shapes of edge components to identify the presence of ice. To achieve this, the proposed method finds the major and minor axes for each edge component, and then draws parallel lines to the major and minor axes over the edge components. For each parallel line to the major and minor axes, the proposed method further extracts angle and density-based features for pixels that fall on the parallel lines to the major and minor axes. Next, the proposed method selects features from each average wavelet image and further calculates the mean for the feature vectors corresponding to the level, which results in a feature matrix. Finally, the feature matrix is fed to a Multi-Layer Perceptron Neural Network for classifying ice and non-ice images. Experimental results on a diversified dataset and comparative study with an existing method show that the proposed method is useful for accurate ice detection with better accuracy.
\end{abstract}

Keywords: Wavelet decomposition, Canny edge components, Major axis, Angle, Density and Multilayer perceptron neural network.

\section{Introduction}

Due to unexpected climate changes, it is hard to predict the impact of natural disasters on social lives in the world (Romeo, 2013; Zhang, 2019). One such disaster is the formation of ice on power 
transmission lines, which is a problem growing considerably due to prevailing macroclimate, micrometeorological and microtopographical conditions (Wang, 2017). Therefore, it can cause many serious accidents, such as short-circuit, grounding, wire breakage, tower toppling, flashover, communication interruption and a great threat to the power grid. This results in national economic loss and a large reduction in productivity (Zeng, 2012). This is a global challenge for many countries, namely, China, Norway, Canada, Russia, Japan and other nations. Hence, one can understand that the problem is sensitive and requires an intelligent and expert system approach that detects ice in images accurately. To find a solution to the above challenges, there have been methods developed for monitoring ice coverage of power transmission lines, ice thickness identification, and monitoring the condition of power cables in the literature ( $\mathrm{Li}, 2015$; Jiao, 2010). However, the methods only perform well for images containing a good amount of ice covering the power transmission lines. For example, sample images with ice can be seen in Fig. 1(a), where ice is visible in all the images with a certain amount. In reality, it is not true because one can expect images which appear as ice, such as images with snow, power lines without ice, fog, white colored objects, etc. For instance, sample images without ice are shown in Fig. 1(b), where it can be seen that images contain power lines without much ice coverage, power lines without ice, and ice cubes without power lines. In this work, we consider the sample images shown in Fig. 1(b) as non-ice images.

When the input is mixed with the above mentioned images without ice, the performance of the existing methods degrade substantially. It is evident from the illustration shown in Fig. 2(a)-Fig. 2(b), where we can see that the performance of the ice detection method (Jiao, 2010) is better for images after classification compared to those before classification. In Fig. 2(a) and Fig. 2(b), for the first image, the method works well for both before and after classification. However, for the second and third images, the method does not work well. This leads to poor results for ice detection and monitoring methods. The ice detection method proposed in Jiao (2010) works based on the use of support vector machines and mathematical morphology. This is justifiable because after we classify images, an existing method can be tuned and modified according to the content of the images, which in turn improves the performance of the method. In other words, classification helps in reducing the complexity of the problem. Therefore, it is necessary for the classification of images that have ice covering power transmission lines. Hence, this work focuses on developing a new expert system for the classification of ice and non-ice images such that existing methods can be used or modified for enhancing the performance of both ice thickness detection and ice monitoring. However, as shown in Fig. 1(b), the content in non-ice images appear as ice in several situations. In addition, the shapes of ice and the background complexity are not predictable as shown in Fig. 2(a), where we can see variations of the background for all the images. These factors make the problem much more challenging and interesting. 

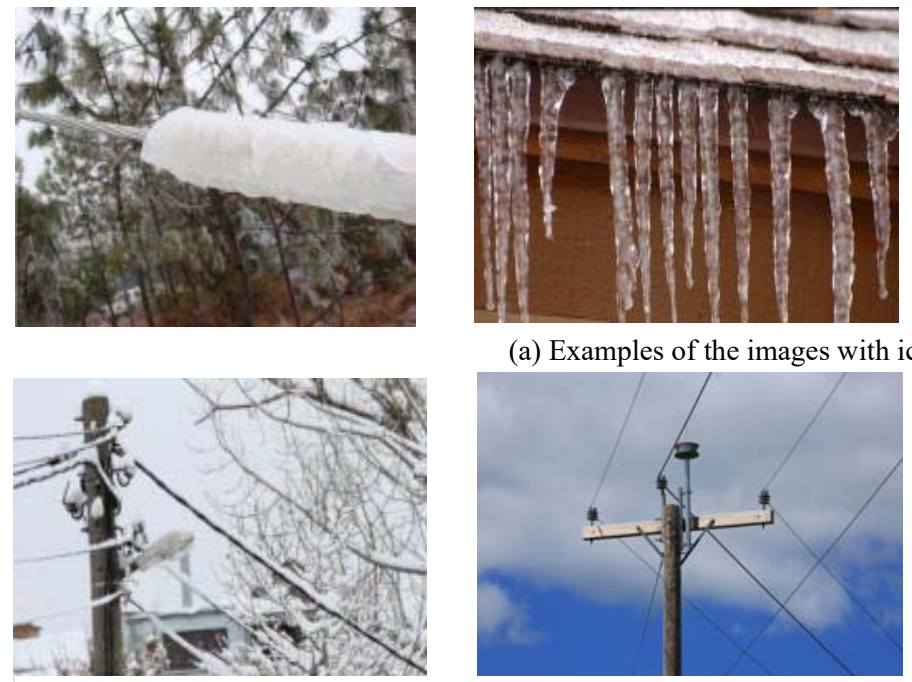

(b) Examples of the images without ice

Fig. 1. Sample ice and non-ice images for classification.
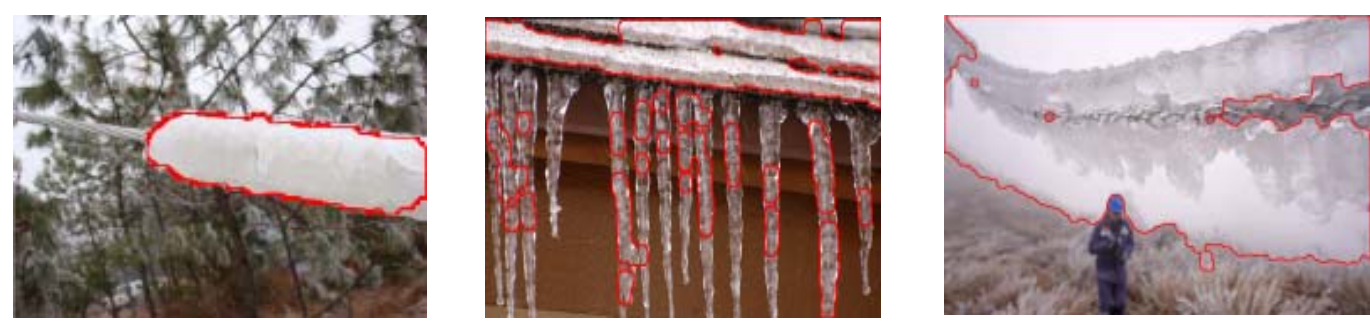

(a) Ice detection by the method in [6] before classification
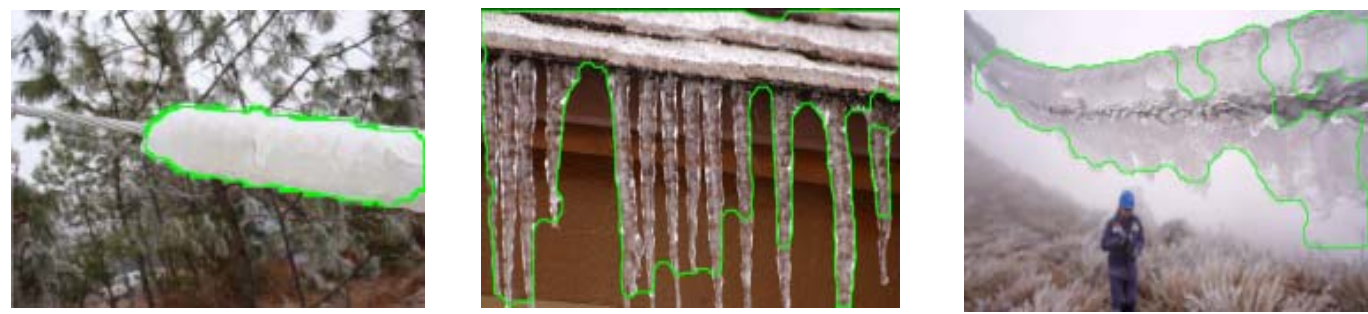

(b) Ice detection by the method in [6] after classification

Fig. 2. Performance of ice detection before and after classification

\section{Related Work}

There are methods for ice thickness identification, monitoring for ice covering of power transmission lines, ice recognition, etc. Most of these methods in the literature address the above-mentioned issues but not the classification of ice and non-ice images. Therefore, in this section, we review the methods of ice thickness identification, detection, monitoring and recognition.

Jiao (2010) proposed the detection of encased ice on transmission lines based on SVMs and mathematical morphology. This method explores an SVM classifier for the classification of pixels that represent ice and non-ice, respectively. Shape features are extracted using morphological operations for eliminating false positives to improve results. The method works well for images having a certain amount of ice with shapes. 
Li (2015) proposed ice detection on electrical power cables. The method explores a neural network classifier for the classification of ice regions in images. It extracts different texture and statistical features, which are fed to a neural network for classification. The method is good for high-contrast and high-resolution images as it depends on edge information. Zeng (2012) proposed a novel thickness detection method of ice covering on overhead transmission lines. This method does not consider images as the input for ice thickness detection, rather it uses temperature coefficients, compressive conductors, wind pressure, etc. As long as the devices send accurate information, the method works well. However, the method may not detect the exact location of ice in the area.

Li (2015) proposed icing thickness detection of high voltage transmission lines based on machine vision. In this method, a hardware device is calibrated to find the relationship between the distance from the observation point to the object and the geometric size of each pixel. Then it extracts edges and a number of pixels to detect ice thickness in the image. The performance of the method depends on the hardware device. When the weather changes drastically, the method may not perform well.

Chang (2018) proposed a study on the calculation of ice thickness and wire extraction based on infrared images. The method uses infrared images as the input for ice thickness detection. It extracts Canny edges for infrared images, and then the Hough transform is applied to find the thickness of the ice. For images with complex backgrounds, the method may not perform well due to the Canny edge operator. The Canny edge operation is sensitive to complex backgrounds.

Zhong (2013) proposed a new image-based algorithm for ice detection and icing thickness estimation for transmission lines. The method explores edge information in images, and then presents a calibration method for estimating ice thickness. It traces the transmission lines to detect ice in the images. However, the method considers images with ice as the input for ice thickness estimation. If we feed images with any object that looks like ice, the method does not perform well. Liu (2017) proposed the detection of ice thickness for high voltage transmission lines by image processing. The method uses histogram equalization for enhancing the information in the images. It extracts edge information for the enhanced images, and then uses the Hough transform for detecting ice thickness. However, the method is developed for images of specific areas and regions in China.

Zhao (2018) proposed an ice monitoring system for transmission lines based on images and stresses. The method considers that icing is a two-class problem, and classifies the normal state for non-ice and the abnormal state for ice. Further, the method uses a recognition algorithm based on pixel changes for ice monitoring. Though the method works for real-time environments, it does not give a perfect solution for different situations, where ice images have complex backgrounds.

Sun (2018) proposed image processing and recognition technologies for transmission line ice-related research. The method explores OTSU's thresholding algorithm for binarizing images, and then uses connected component analysis for ice-transmission line detection. It is true that Otsu's thresholding works well for images with simple backgrounds but not complex backgrounds such as our dataset. Yang (2017) proposed image recognition for determining ice thickness on power transmission lines based on fractal edge detection. The method uses blanket dimension fractals and the discontinuous edges of ice for estimating ice thickness. Furthermore, a calibration coefficient is obtained according to the relationship between pixel values and the actual values of power transmission lines. However, since edges are sensitive to complex backgrounds, the performance of the method may degrade for images without ice. 
Wang (2017) proposed image recognition for determining ice thickness on power transmission lines based on least squares and the Hough transform. The method explores Canny edges, and then combines the Hough transform with least squares for ice identification in images. Thickness is estimated based on a geometric calculation model, which provides the radius of power lines. The method is said to work well for images with complex backgrounds and different intensity levels. However, it is unclear how the method takes care of images without ice regions.

In light of the above discussions, it is noted that most of the methods focus on ice thickness detection, monitoring and recognition with different ideas. It shows that the existing methods address several challenges of ice thickness detection. However, none of the methods address the issue caused by images without ice regions. Images without ice regions and objects in images that appear as ice are common in places of low temperature, snow and fog. In this context, existing methods lose the ability to deal with these situations, which leads to poor results. Since the issue is sensitive and has a huge impact on society and globally, accurate results are important. Therefore, to increase the performance of ice detection or thickness estimation and monitoring methods, there is a need for the classification of ice and non-ice images before detection and recognition.

Hence, in this work, we aim at developing a new expert system for the classification of images containing ice and without ice. Since the size of ice thickness varies and is not predictable, we explore Haar wavelet decomposition to overcome the issue of size. This is because, as the number of decomposition levels increases, the method can provide finer details in images despite changes in the size of ice thickness (Liang, 2017). It is true that if images contain ice, one can expect unique directions and dense pixels at ice regions. However, this is not true for images without ice, where we can expect dense and some arbitrary shapes but not in a unique direction. It is evident from the literature that Canny edge information provides prominent information for ice detection. Inspired by this observation, we estimate the major and minor axes for each edge component in images at the decomposition levels. Angle and density for pixels that fall on parallel lines to the major and minor axes are extracted to study the shapes of ice regions, which results in a feature matrix. The feature matrix is then fed to Multi-Layer Perceptron (MLP) Neural Network (NN) for the classification of ice and non-ice images (Raghu, 2017; Zhao, 2015). The key contributions of the proposed work are: (1) Use of wavelet decomposition for undertaking Candidate Component Detection (CCD) from the input image, and (2) The way the proposed method combines angle and density features for studying shapes of ice in images irrespective of rotations and scaling.

\section{The Proposed Methodology}

As mentioned in the previous section, for each input image, the proposed method obtains high frequency sub-bands, namely, LH, HL and HH for four levels of wavelet decomposition. Since input images suffer from low resolution and contrast, to enhance the details, the proposed method generates average images by adding LH, HL and HH high frequency sub-bands, which outputs four average images for the respective four levels of decomposition. The proposed method then obtains Canny edge images for the four average images. In order to smooth the edge information, the proposed method performs a singlepixel gap-filling morphological operation, which results in Candidate Components Detection (CCD). For each component in the CCD image of four levels, the proposed method finds the major and minor axes, and then estimates the angle and density for pixels that fall on parallel lines to the major and minor axes in order to study the presence of ice. To reduce the number of computations, the proposed method 
considers the top 20 angles and density values corresponding to those lines parallel to the major and minor axes of the four wavelet decomposition levels. Furthermore, the proposed method finds the mean for the top 20 values corresponding to four levels, which results in a feature matrix with dimensions of $2 \times 40$. This includes two feature vectors having 20 angle-based features and 20 density-based features for each input image, respectively. We believe that the proposed features represent the unique properties of ice and non-ice images. Besides, as stated in (Nanni, 2018) that the combination of feature extraction and learning gives better results than learning with raw pixels. Furthermore, Multi-Layer Perceptron (MLP) Neural Networks (NNs) have the ability to handle non-linear data for classification. Therefore, we propose to explore MLP-NNs for the classification of ice and non-ice images rather than exploring deep learning with raw pixels in this work. The whole framework of the proposed method is shown in Fig. 3, where we can find the flow and logic of the proposed method. In this work, we consider images containing power lines without ice, images with snow or fog, and images having ice without power lines as non-ice images for experimentation.

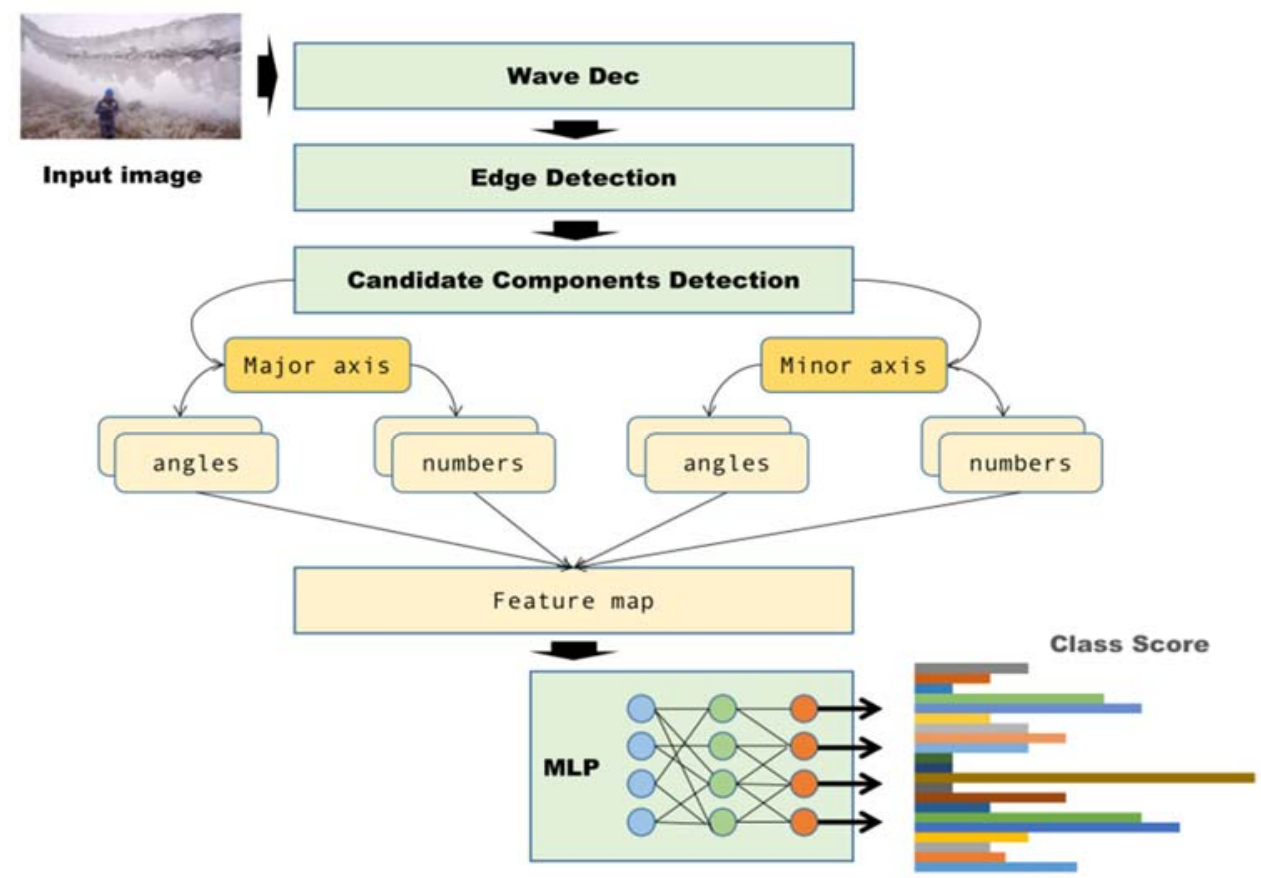

Fig. 3. The Framework of the proposed method

\subsection{Candidate Components Detection}

For each input image of the first level of Haar wavelet decomposition, the proposed method obtains three high frequency sub-bands as shown in Fig. 4(a) and Fig. 4(b) for ice images and non-ice images, respectively. It is noted from Fig. 4(a) and Fig. 4(b) that LH, HL and HH highlight the prominent information of horizontal, vertical and diagonal directions, respectively. In order to take advantage of LH, HL and HH, the proposed method computes an average for LH, HL, HH sub-bands at the pixel level as shown in Fig. 4(a) and Fig. 4(b) for ice and non-ice images, respectively, where it is observed that the fine details in the average images are enhanced compared to the high frequency sub-bands. This process continues for all the four levels of wavelet decomposition as shown in Fig. 5(a) and Fig. 5(b) for ice and non-ice images, where one can see the average images for the four levels. In Fig. 5(a) and 
Fig. 5(b), it is noted that as the number of levels increases, the components in the images get clear shapes in the case of ice images, while in the case of non-ice images, the shapes of the components are arbitrary. This is the advantage of wavelet decomposition and the average of high frequency sub-bands. The mathematical formulation for Haar wavelet decomposition, namely, LH, HL, HH, and the average of high frequency sub-bands, are defined in Equation (1) to Equation (10).

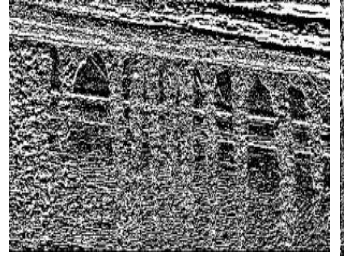

LH

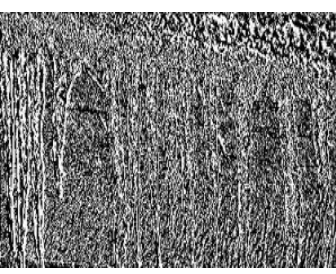

HL

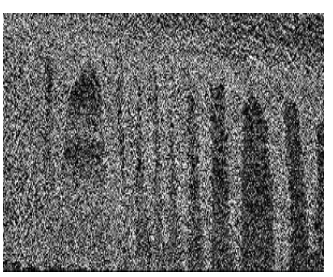

$\mathrm{HH}$

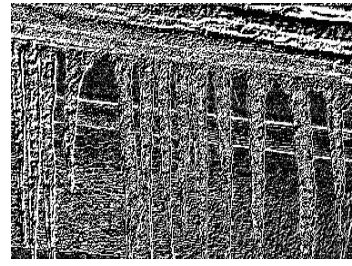

Average

(a) Wavelet high frequency sub-bands of the Ice image (second image in top row)

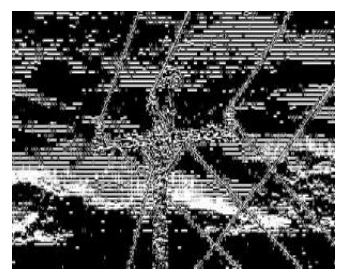

LH

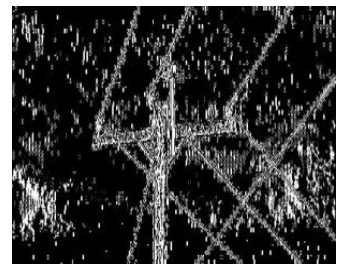

HL

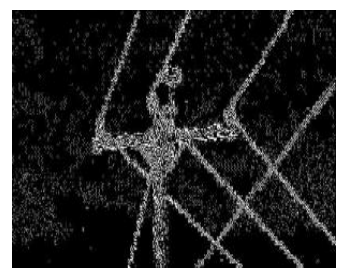

$\mathrm{HH}$

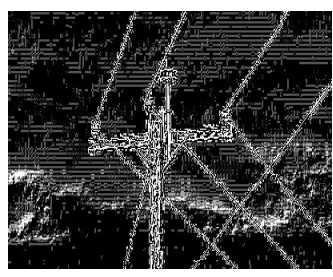

Average

(b) Wavelet high frequency sub-bands of non-Ice image (second image in bottom row)

Fig. 4. Enhancing fine details in the image using Wavelet decomposition

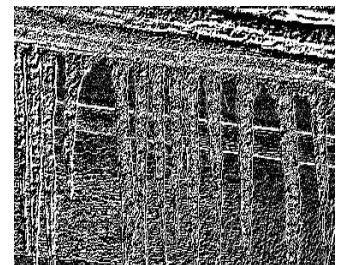

Level-1

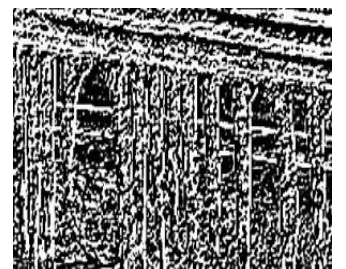

Level-2

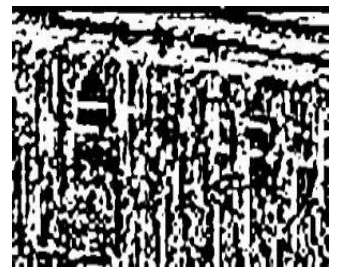

Level-3

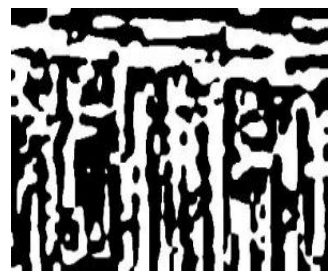

Level-4

(a) Average of high frequency wavelet sub-bands of ice image for level-1 to level-4

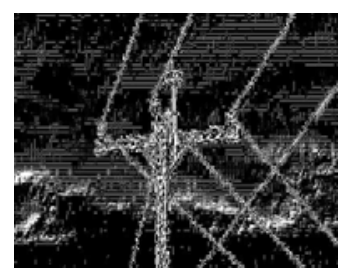

Level-1

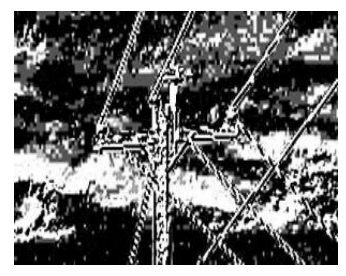

Level-2

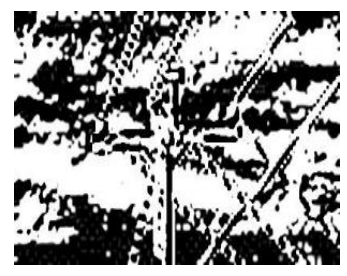

Level-3

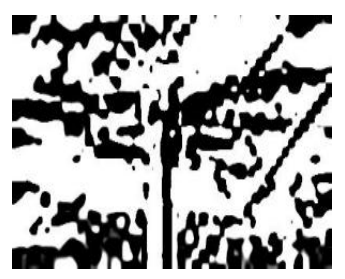

Level-4

(b) Average of high frequency wavelet sub-bands of non-ice image for level-1 to level-4

Fig. 5. Average of high frequency sub-bands of wavelets for multi-levels of decomposition.

$$
\phi(x)= \begin{cases}1 & 0 \leq x<1 \\ 0 & \text { otherwise }\end{cases}
$$




$$
\psi(\mathrm{x})=\left\{\begin{array}{l}
10 \leq x<\frac{1}{2} \\
-1 \frac{1}{2} \leq x<1 \\
0 \text { otherwise }
\end{array}\right.
$$

where $\Phi(x)$ and $\Psi(x)$ are Haar wavelet basis function and its scaling wavelet function, respectively. The 2D scaling function $\Phi(x, y)$ and three 2D wavelet functions are defined below.

$$
\begin{gathered}
\phi(\mathrm{x}, \mathrm{y})=\phi(\mathrm{x}) \phi(\mathrm{y}) \\
\psi^{\mathrm{LH}}(x, y)=\psi(x) \phi(y) \\
\psi^{\mathrm{HL}}(x, y)=\phi(x) \psi(y) \\
\psi^{\mathrm{HH}}(x, y)=\psi(x) \psi(x, y)
\end{gathered}
$$

where LH, HL and HH denote, horizontal, vertical and diagonal direction sub-bands, respectively.

$$
\begin{gathered}
\mathrm{LH}=\frac{1}{\sqrt{M N}} \sum_{1}^{M} \sum_{1}^{N} I(x, y) \psi^{L H}(x, y) \\
\mathrm{HL}=\frac{1}{\sqrt{M N}} \sum_{1}^{M} \sum_{1}^{N} I(x, y) \psi^{H L}(x, y) \\
\mathrm{HH}=\frac{1}{\sqrt{M N}} \sum_{1}^{M} \sum_{1}^{N} I(x, y) \psi^{H H}(x, y) \\
\text { AVER }=\frac{L H+H L+H H}{3}
\end{gathered}
$$

where $\mathrm{M}$ an $\mathrm{N}$ denote the sizes of an image, and AVER denotes the average of the three high frequency sub-bands.

As mentioned above, to study the shapes of the components, we propose to obtain Canny edges for the average of the four levels of wavelet decomposition as shown in Fig. 6(a) and Fig. 6(b), for ice and nonice images, respectively. It is clear from the results in Fig. 6(a) and Fig. 6(b) that the shapes of components in all the four levels appear better compared to the results in Fig. 5(a) and Fig. 5(b). Due to the complex background and foreground variations, sometimes, one can expect disconnections in the edge images. To overcome this problem, the proposed method performs a single pixel morphological operation over Canny edge images, which fills the gap as shown in Fig. 7(a) and Fig. 7(b) for both ice and non-ice images. This results in Candidate Component Detection (CCD). 


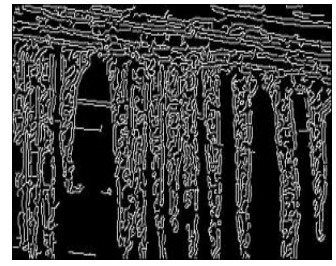

Level-1

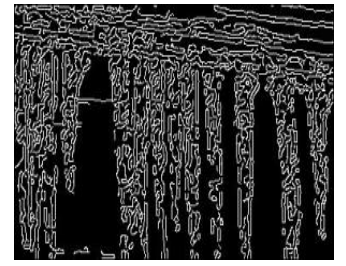

Level-2

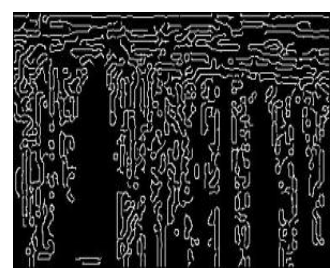

Level-3

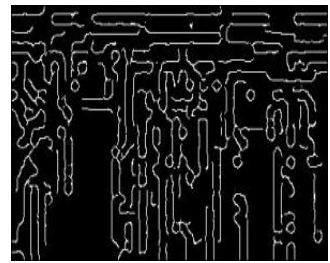

Level-4

(a) Canny edge components for the average of high frequency sub-bands of an ice image

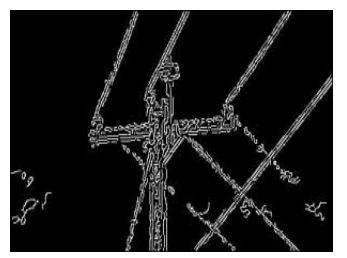

Level-1

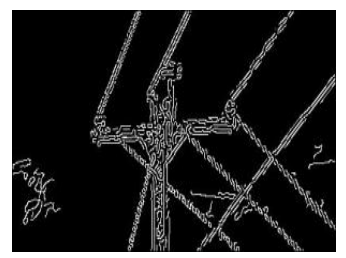

Level-2

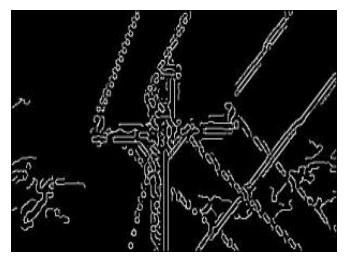

Level-3

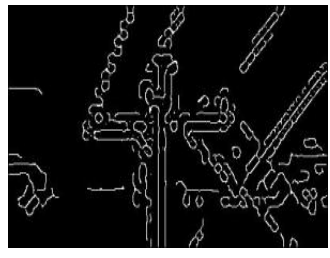

Level-4

(b) Canny edge components for the average of high frequency sub-bands of a non-ice image.

Fig. 6. Edge components detection for Ice and non-Ice images of average of high frequency of sub-bands

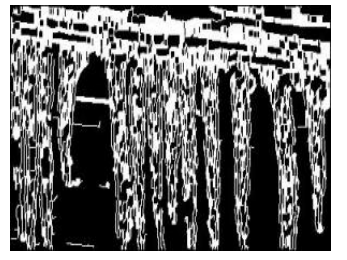

Level-1

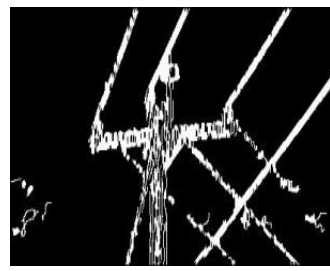

Level-1

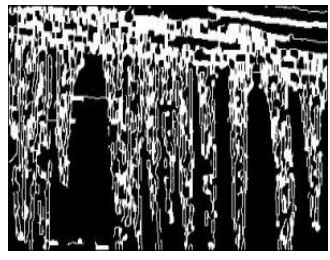

Level-2

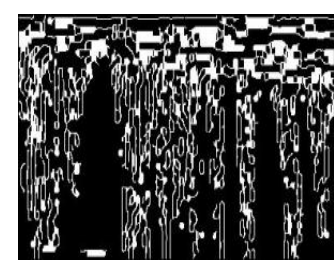

Level-3

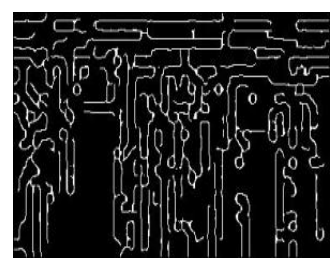

Level-4

(a) Morphological operation on the ice image

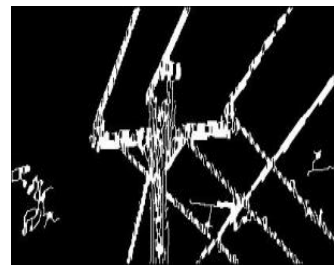

Level-2

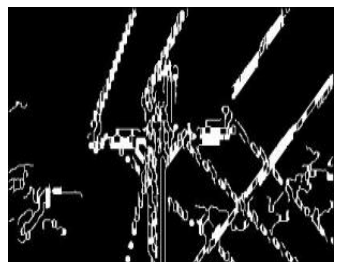

Level-3

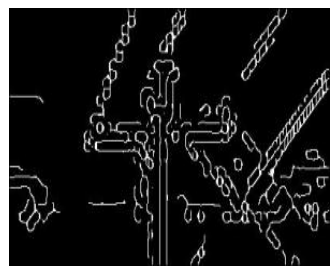

Level-4

(b) Morphological operation on the non-ice image

Fig. 7. Morphological operations for filling one or two pixel gaps (candidate components detection).

\subsection{Angle and Density-based Features for Ice Image Classification}

For each component in CCD images of the four-wavelet decomposition levels, the proposed method draws the major and minor axes using $\mathrm{X}$ and $\mathrm{Y}$ coordinates of pixels in components as defined in Equation (11). It is illustrated in Fig. 8(a) and Fig. 8(b), respective, for ice and non-ice images, where we can see blue lines denote the major axis, and the green lines denote the minor axis. The line perpendicular to the major axis with the minimum distance is considered as the minor axis. The proposed method finds parallel lines to the major and minor axes for candidate components. For each parallel line, the proposed method calculates angle and counts the number of pixels as the density for the pixels that fall on parallel lines to the major and minor axes, which results in angle and density- 
based features. The processes of angle and density estimation for the sample components in ice and non-ice images are illustrated in Fig. 9(a) and Fig. 9(b), respectively. In Fig. 9(a) and Fig. 9(b), we can clearly see parallel lines to the major axis (red color) are drawn represented by a blue color, and parallel lines drawn to the minor axis (red color) are represented by a green color in both candidate components of ice and non-ice images.

$$
\mathrm{L}_{\text {major }}=\tan \theta *\left(x-O_{x}\right)+O_{y}, \mathrm{~L}_{\text {minor }}=\cot \theta *\left(x-O_{x}\right)+O_{y}
$$

where $(\mathrm{Ox}, \mathrm{Oy})$ denotes centroid coordinates of the component from which the ellipse is drawn. $\theta$ represents the angle between the major/minor axis and the $\mathrm{X}$-axis.

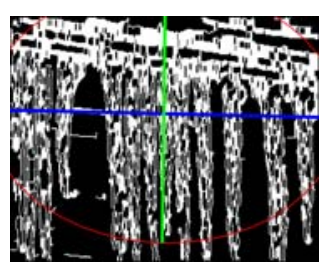

Level-1

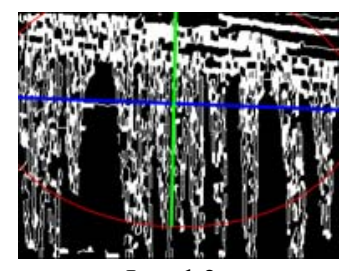

Level-2

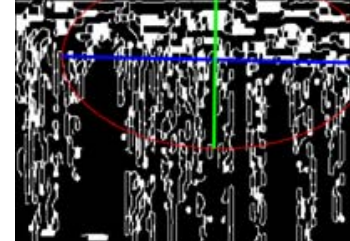

Level-3

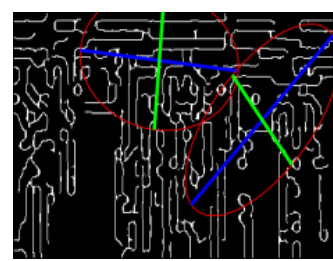

Level-4

(a) Major and minor axes for each candidate components in ice images

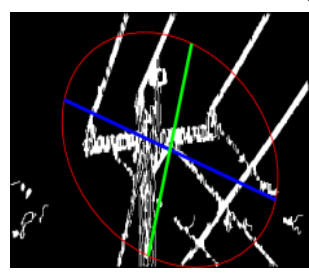

Level-1

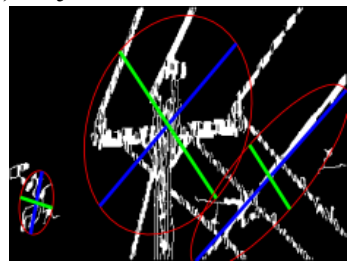

Level-2

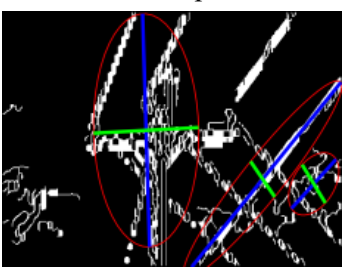

Level-3

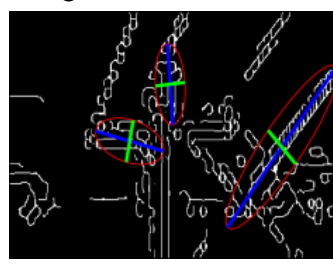

Level-4

(b) Major and minor axes for each candidate components in non-ice images

Fig. 8. Drawing major and minor axes for the candidate components. Blue lines and red lines denote the major axis and minor axes, respectively.

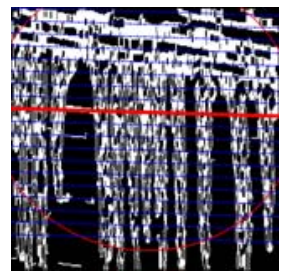

(a) Major axis

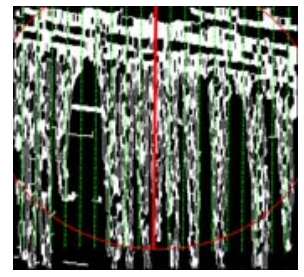

Minor axis-ice image

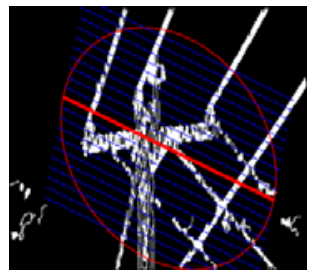

(b) Major axis

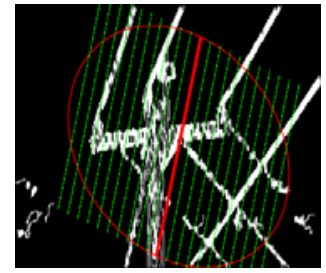

Minor axis-non ice image

Fig. 9. Angle and Density feature extraction for parallel lines to the major and minor axes over the candidate components in Ice and non-ice images

To reduce the number of computations, the proposed method selects the top 20 angles as defined in Equation (12), and density values from each level, which includes parallel lines to both the major and minor axes. The value of 20 is determined empirically based on predefined samples chosen randomly from our database. Further, the proposed method calculates the mean of the top 20 angle values corresponding to four levels, which gives a feature vector containing 20 angle values for each image. In the same way, the proposed method estimates density for the components in the four levels, which results in a feature vector containing 20 density-based features for each image. Therefore, the proposed 
method obtains a feature matrix of size $2 \times 40$ dimension for each input image as defined in Equation (13) and Equation (14). The extracted features are invariant to rotation and scaling because they follow angles of the major/minor axes of the components and use multi-levels of wavelet decomposition for reducing the effects of different scaling. Therefore, different rotations and scaling do not affect the performance of the proposed method. The effect of angle and density-based features for ice and nonice images are illustrated in Fig. 10 and Fig. 11, respectively, for all the four levels, where graphs are drawn for 20 parallel lines to the major and minor axes vs angle and density feature values. Fig. 10 shows that as the number of levels increases, the number of peaks also increases for ice images as shown in Fig. 10(a) and Fig. 10(b), while the graph behaviors in Fig. 10(c) and Fig. 10(d) of non-ice images are arbitrary. Similarly, the proposed method draws graphs for the density feature as shown in Fig. 11(a)-Fig. 11(d), where we note that the graph behaviors with respect to rows and columns of ice images are almost constant, while the behaviors are inconsistent for non-ice images.

$$
\mathrm{a}=\arctan \left(\frac{\sum\left(\mathrm{x}_{\mathrm{i}}-\bar{x}\right)\left(\mathrm{y}_{\mathrm{i}}-\bar{y}\right)}{\sum\left(x_{i}-\bar{x}\right)^{2}}\right)
$$

where $\left\{\left(x_{i}, y_{i}\right) \mid\left(x_{i}, y_{i}\right) \in\right.$ component $\}$ denotes all the white pixels of each parallel line, where $i$ varies from 1 to $n$. Here, $n$ is the number of pixels in each parallel lines.

$$
\text { feature }=\left[\begin{array}{c}
\Lambda\left(\mathrm{A}_{\text {row }}\right) \\
\Lambda\left(A_{\text {column }}\right) \\
\Lambda\left(N_{\text {row }}\right) \\
\Lambda\left(N_{\text {column }}\right)
\end{array}\right]
$$

where $\mathrm{N}\left[n_{1}, \ldots, n_{20}\right]$ represents a vector containing the mean of top 20 density feature values of the four levels. $\mathrm{A}\left[a_{1}, \ldots, \mathrm{a}_{20}\right]$ represents the vector containing the mean of the angle feature values of the four levels. $\Lambda()$ denotes the function for feature extraction.

$$
\text { feature }=\left[\begin{array}{l}
\Lambda\left(\frac{1}{M}\left(\mathrm{~A}_{1_{\mathrm{r}}}+\mathrm{A}_{2_{\mathrm{r}}}+\cdots+\mathrm{A}_{\mathrm{M}_{\mathrm{r}}}\right)\right) \\
\Lambda\left(\frac{1}{M}\left(\mathrm{~A}_{1_{\mathrm{c}}}+\mathrm{A}_{2_{\mathrm{c}}}+\cdots+\mathrm{A}_{\mathrm{M}_{\mathrm{c}}}\right)\right) \\
\Lambda\left(\frac{1}{M}\left(\mathrm{~N}_{1_{\mathrm{r}}}+\mathrm{N}_{2_{\mathrm{r}}}+\cdots+\mathrm{N}_{\mathrm{M}_{\mathrm{r}}}\right)\right) \\
\Lambda\left(\frac{1}{M}\left(\mathrm{~N}_{1_{\mathrm{c}}}+\mathrm{N}_{2_{\mathrm{c}}}+\cdots+\mathrm{N}_{\mathrm{M}_{\mathrm{c}}}\right)\right)
\end{array}\right]
$$

Here $A_{i_{r}}$ denotes the vector of row angles in the $i$-th component, and $A_{i_{c}}$ denotes the vector of column angles in the $i$-th component. Note that $\mathrm{N}_{\mathrm{i}_{\mathrm{r}}}$ and $\mathrm{N}_{\mathrm{i}_{\mathrm{c}}}$ are the same. 
Level-1
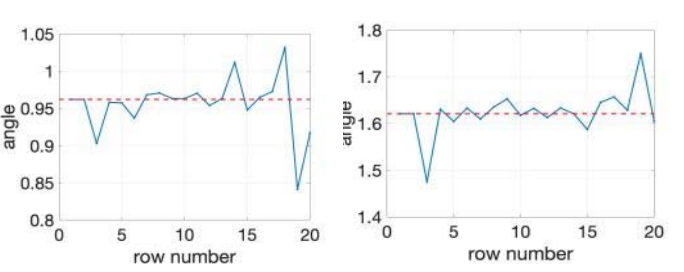

(a) Histograms for
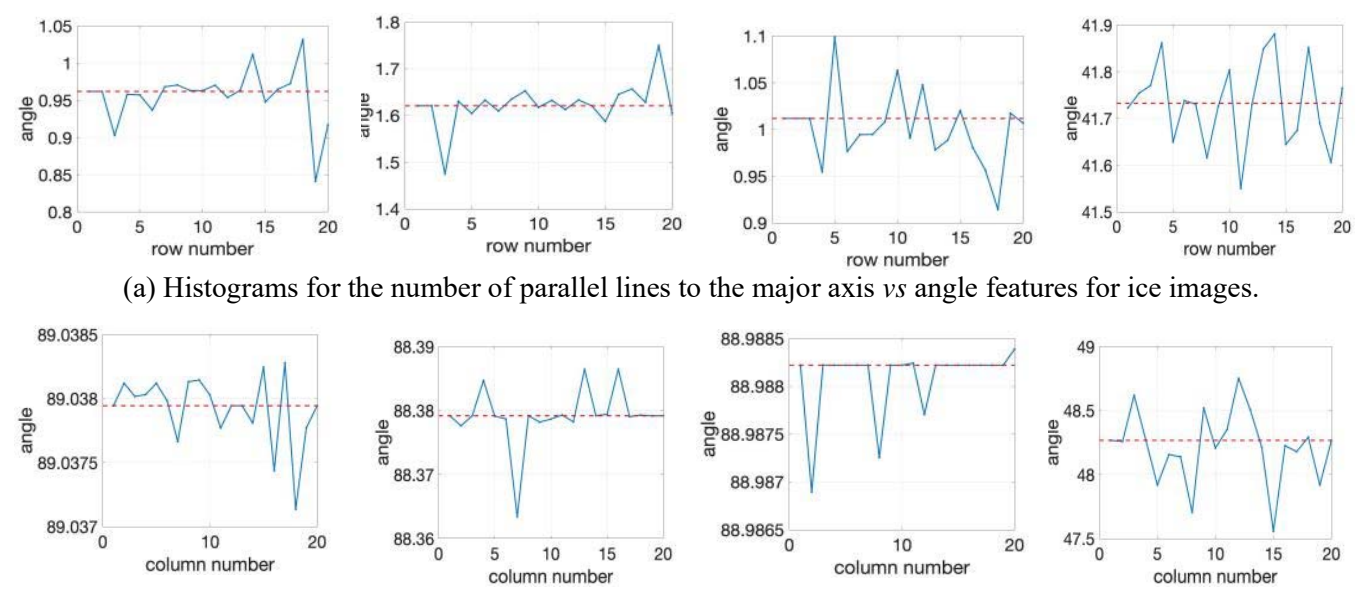

(b) Histograms for the number of parallel lines to the minor axis vs angle features for ice images.
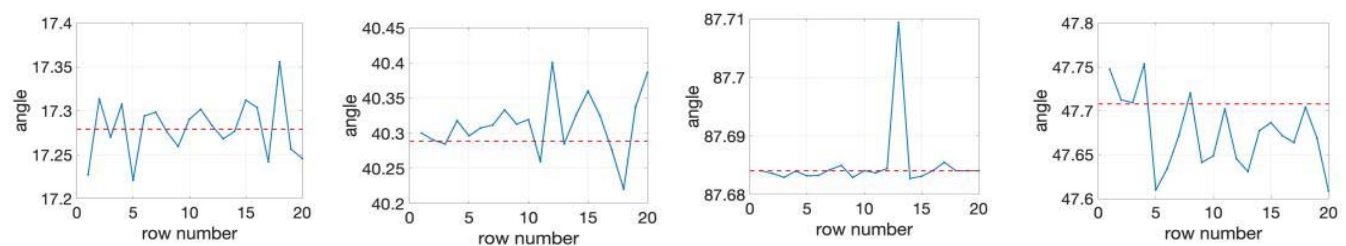

(c) Histograms for the number of parallel lines to the major axis vs angle features for non-ice images.
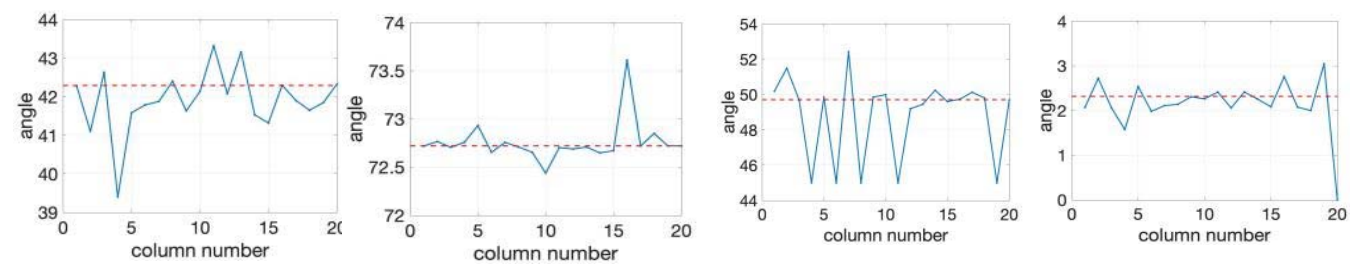

(d) Histograms for the number of parallel lines to the minor axis vs angle features for non-ice images.

Fig. 10. Histogram representation for angle-based features for ice and non-ice images. 

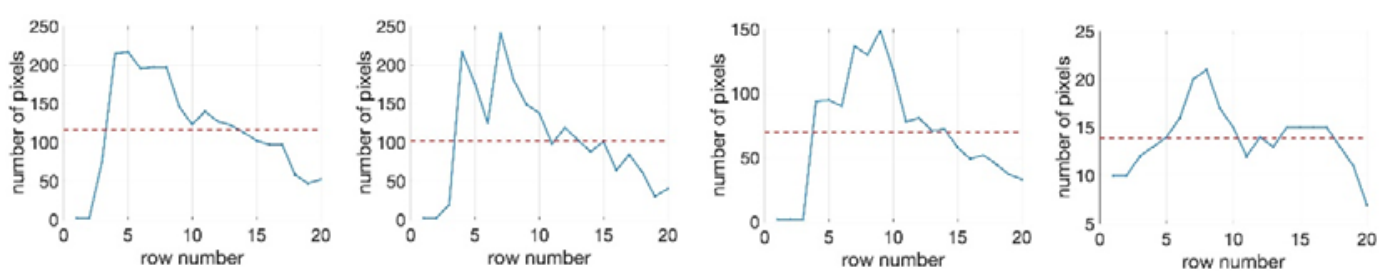

(a) Histograms for the number of parallel lines to the major axis vs dense features for ice images.
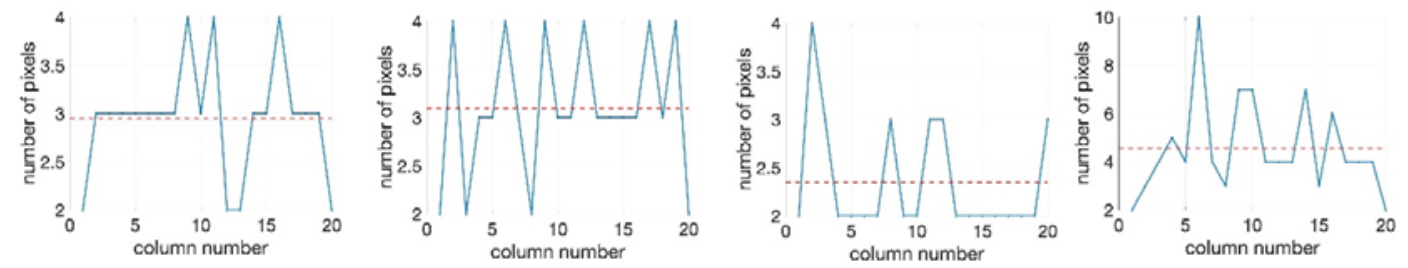

(b) Histograms for the number of parallel lines to the minor axis $v s$ dense features for ice images.
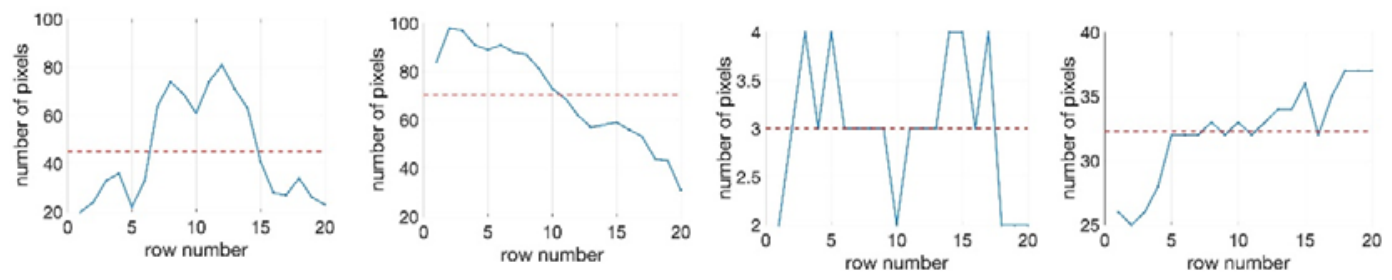

(c) Histograms for the number of parallel lines to the major axis $v s$ dense features for non-ice images.
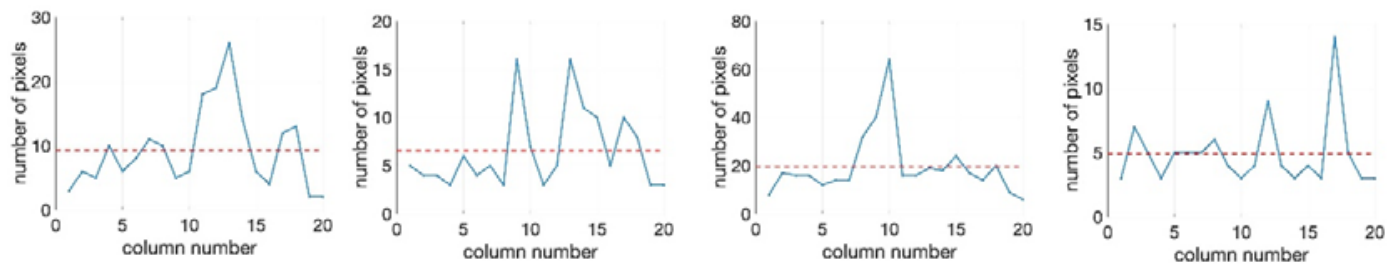

(d) Histograms for the number of parallel lines to the minor axis $v s$ dense features for non-ice images.

Fig. 11. Histogram representation of dense-based features for ice and non-ice images.

Finally, the feature matrix obtained is passed to a Multi-Layer Perceptron (MLP) Neural Network (MN) for classification. The MLP-NN is a feed forward neural network consisting of an input layer, a hidden layer and an output layer. MLP makes use of a supervised learning technique called back propagation for training the network, which has been used in this work as a binary classifier. For choosing the number of samples to train and test the classifier, we follow 10-fold cross-validation in this work for all the experiments. The parameters that we use for classification are as follows. We set 0.3 for LR, 0.2 for momentum, and the hidden-layers are (attributes + classes) $/ 2$, the batch-size is 100 and the remainder are default parameters as in the method of (Raghu, 2017), where one can find more details about the MLP-NN for classification.

\section{Experimental Results}

Despite several methods proposed for ice detection, thickness identification and monitoring, there are no standard datasets available in the literature. The main reason relates to copyright issues with industry. 
Therefore, we create our own dataset by capturing ice covered power transmission lines, and also from internet sources. As a result, our dataset consists of 103 ice images and 101 non-ice images, which gives a total of 204 images for experimentation in this work. Though the size of the dataset is modest, it is effective, as it includes all possible ice images, which generally cause disasters to the best of our knowledge, and are pertinent for experts of the concerned department. The collected ice images include ice coverings of different power transmission lines. Similarly, non-ice images include fog, snow, power lines without ice cover, ice without power transmission lines, ice in the sea, etc. In addition, images of both the classes have no constraints on the background complexity as shown in Fig. 12 (a) and Fig. 12(b), where one can note that images of both classes appear similar from the naked eye, but the content in the images look different. Images of the non-ice class may be confused with those of the ice class, hence there is a high chance of considering the latter as ice images in ice detection, thickness identification, etc. Therefore, it is necessary to classify non-ice images from the list of input ice images for improving the performance of ice detection, thickness identification and monitoring methods.

To measure the performance of the proposed method, we use standard measures, namely, a confusion matrix with an Average Classification Rate (ACR), for the classification of ice and non-ice images. The average classification rate is defined as the mean of diagonal elements of the confusion matrix. We also provide one-more experimental study to validate the proposed classification in this work, that is, ice detection experiments before classification (prior to classification) and after classification. The beforeclassification experiments consider images of both ice and non-ice classes as the input for calculating measures. In the case of after-classification, the proposed method considers individual classes as the input for calculating measures. For ice detection experiments of before- and after- classifications, we use the standard measures, namely, Recall, Precision and F-score as defined in Equations (15)- (17), respectively. In Equations (14)- (17), $\mathrm{C}_{1}$ is the number of images labeled correctly and belongs to the positive class, $\mathrm{C}_{2}$ is the number of images labeled correctly and belongs to the negative class, while $\mathrm{W}_{1}$ and $\mathrm{W}_{2}$ are the numbers of images labeled incorrectly in positive and negative classes, respectively.
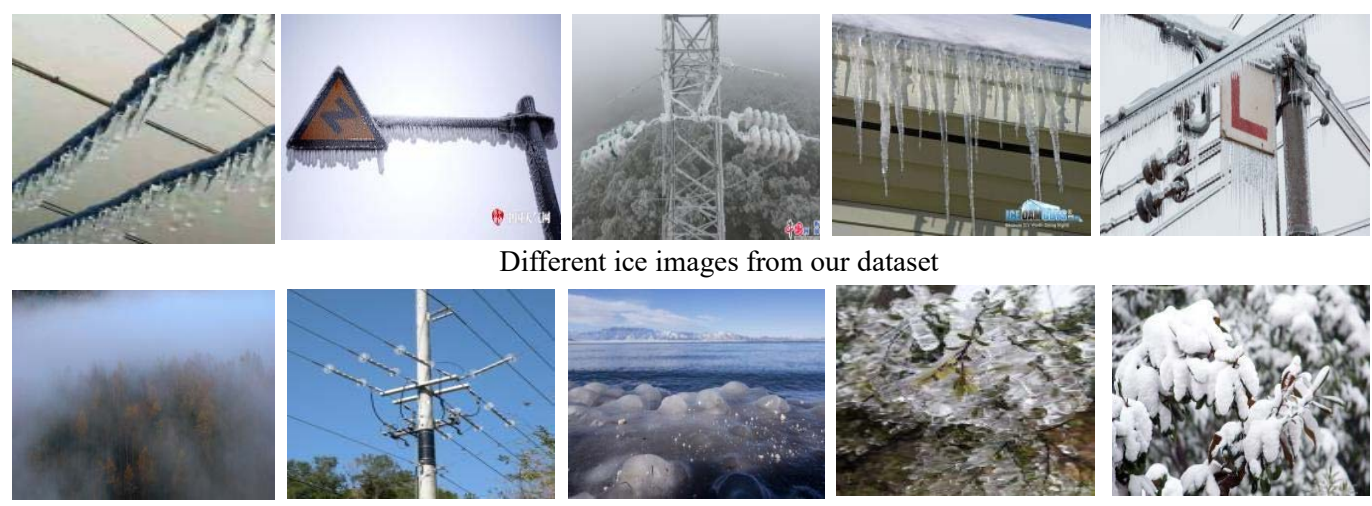

Different ice images from our dataset
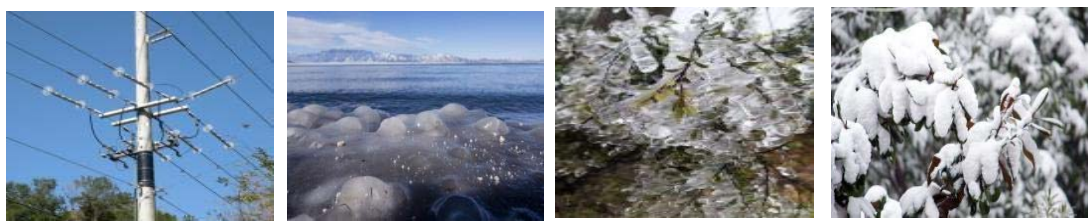

Different non-ice images from our dataset

Fig. 12. Sample images for ice and non-ice classification experiments chosen from our dataset.

$$
\begin{aligned}
& \text { Percision }=\frac{C_{1}}{C_{1}+W_{1}} \\
& \text { Recall }=\frac{C_{1}}{C_{1}+W_{2}}
\end{aligned}
$$




$$
F-\text { Score }=\frac{2 \times(\text { Precision } \times \text { Recall })}{(\text { Precision }+ \text { Recall })}
$$

To show the superiority of the proposed classification method, we implement an existing method from Li (2015), which explores different texture, statistical features and neural networks for the classification of ice and non-ice regions in images. Since the method uses the classification of ice and non-ice regions on input images, we use the same idea for the classification of ice and non-ice images in this work. If the existing method finds ice regions in an input image, it is considered as an ice image, else it is considered as a non-ice one. In this way, the existing method is used for a comparative study in this work. The reason to choose this method for comparison is that it extracts general features and uses a neural network classifier for the classification of ice regions as in the proposed method. We believe that the proposed general features in $\operatorname{Li}$ (2015) work well for images of different complexities.

For ice detection experiments on before- and after-classification, we implement the existing method from Jiao (2010), which explores an SVM classifier and mathematical morphology operations for ice detection. The reason to choose this existing method for the experiments is that the method uses raw pixels with a general SVM classifier for the classification of ice regions without extracting specific features. As a result, we believe that the method can work for different images with minimum constraints. Parameters of the SVM classifier are tuned for performing experiments on afterclassification because the class contains only images with ice regions after removing images without ice regions. It is expected that the ice detection method should give better results for after-classification compared to before-classification due to the reduction in complexity of the problem.

In the proposed method, we use wavelet decomposition levels to overcome the issue of the size of ice regions. To determine the number of levels that give better results for the proposed work, we calculate the classification rate for our dataset by varying the number of wavelet decomposition levels as shown in Fig. 13. Fig. 13 shows that for level 4, the proposed method reports the highest classification rate compared to the other levels. It is justifiable because as the number of wavelet decomposition levels increases, the introduction of blur also increases, which leads to the loss of vital information and hence the classification rate decreases in Fig. 13 after level 4. At the same time, if we use fewer levels (less than 4), information becomes insufficient to extract distinct features for the complex problem of classifying ice and non-ice images. Therefore, we found that the four levels of wavelet decomposition are the best for the proposed work.

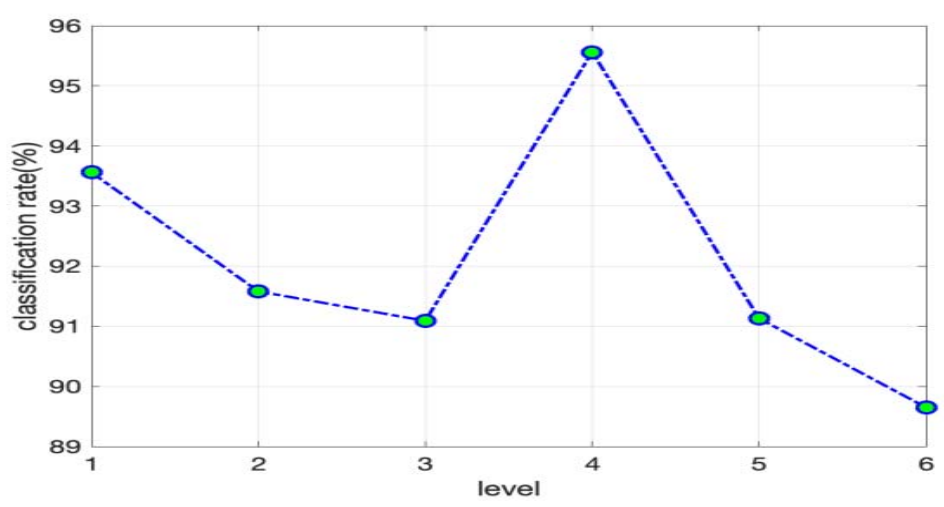

Fig. 13. Determining the number of decomposition levels using our dataset. Number of levels vs classification rate. 
To reduce the number of computations and to balance optimal features, the proposed method chooses the top 20 features from the feature matrix for classification. To validate the value of 20 , we calculate the classification rate on our dataset by varying the number of features as shown in Fig. 14, where it is noted that as the number of features increases, the classification rate also gradually increases. However, at 20, the proposed method reports the highest classification rate, which decreases if we increase the number of features further. Therefore, this shows that the feature of 20 is the best for our dataset.

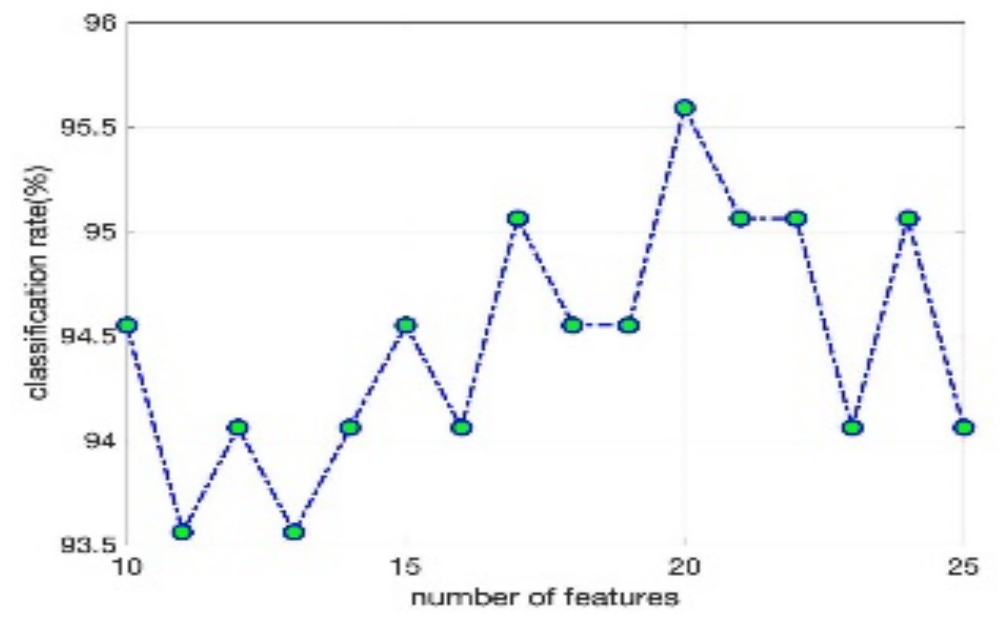

Fig. 14. Determining the top 20 features from the list of features for classification.

\subsection{Experiments on Key Steps of the Proposed Method}

The proposed method consists of a few key steps for achieving better results on the classification of ice and non-ice images, namely, the use of Canny edge images, the major and minor axes, and the average of the top 20 features corresponding to four levels. To assess the contribution of each key step mentioned above, we calculate the classification rate for each experiment with respect to key steps. As noted in the literature, most of the methods use Canny edge images for ice detection, thickness identification and monitoring. This is because Canny edge operators are good for low and high contrast images, while the other existing edge operators may not be good for low contrast images. One such edge operator is the Sobel edge, which works well for high contrast images because the process involves the first order derivative. However, in the case of the proposed work, images with low contrast are common. To validate the statement, we generate a confusion matrix with ACR for the proposed method with Sobel as reported in Table 1, where it is noted that the proposed method with Canny is better than the proposed method with Sobel.

In order to make the proposed method rotation invariant, we extract features for parallel lines to the major and minor axes of candidate components in the image. When we ignore the major and minor axes for feature extraction, the proposed method considers rows and columns instead of parallel lines to the major and minor axes. Since the proposed dataset does not have any constraints on rotations, images can have any rotations. As a result, the features extracted without considering the major and minor axes may not capture distinct properties of candidate components in the image for classification. This leads to poor results for feature extraction. It is evident from the results of the confusion matrix and ACR reported in Table 1 that the proposed method without major and minor axes, score low results compared 
to the proposed method.

To take the advantage of the four-wavelet decomposition levels, the proposed method computes the mean of the feature vectors corresponding to four levels. To test the contribution of average features for the classification of ice and non-ice images, we compute a confusion matrix and ACR without averaging features of the four levels as reported in Table 1. In this experiment, we consider features of four vectors of the respective four levels for experimentation rather than considering one feature vector. It is noted from Table 1 that the proposed method without averaging, scores slightly low results compared to the proposed method with averaging. This shows that there is not much effect on the overall performance of the proposed method. However, if we consider a single feature vector instead of four feature vectors, the proposed method saves a lot on computations.

Table 1. Confusion matrices for assessing the contribution of the key steps of the proposed method (in \%).

\begin{tabular}{|c|c|c|c|c|c|c|c|c|}
\hline Classes & \multicolumn{2}{|c|}{$\begin{array}{c}\text { Proposed Method- } \\
\text { Canny }\end{array}$} & \multicolumn{2}{c|}{$\begin{array}{c}\text { Proposed method- } \\
\text { Sobel }\end{array}$} & \multicolumn{2}{c|}{$\begin{array}{c}\text { Proposed method without } \\
\text { major and minor axis }\end{array}$} & \multicolumn{2}{|c|}{$\begin{array}{c}\text { Proposed method without } \\
\text { averaging density and angle } \\
\text { features }\end{array}$} \\
\cline { 2 - 9 } & Ice & Non-Ice & Ice & Non-ice & Ice & Non-ice & Ice & Non-ice \\
\hline Ice & $99.03 \%$ & $0.97 \%$ & $55.34 \%$ & $44.66 \%$ & $90.29 \%$ & $9.71 \%$ & $95.15 \%$ & $4.85 \%$ \\
\hline $\begin{array}{c}\text { Non- } \\
\text { ice }\end{array}$ & $7.92 \%$ & $92.08 \%$ & $19.80 \%$ & $80.20 \%$ & $13.86 \%$ & $86.14 \%$ & $6.93 \%$ & $93.07 \%$ \\
\hline ACR & \multicolumn{2}{|c|}{95.59} & \multicolumn{2}{|c|}{67.65} & \multicolumn{2}{c|}{88.24} & \multicolumn{2}{c|}{94.12} \\
\hline
\end{tabular}

The proposed method combines angle and density features for the classification of ice and non-ice images in this work. In order to assess the contribution of individual features, we produce a confusion matrix and compute classification rate for only angle-based features and density features, and the results are reported in Table 2. It is confirmed from Table 2 that both angle and density features contribute almost equally to the classification of ice and non-ice images as both the features score almost the same classification rate. Hence, the proposed method that considers both angle and density features reports the highest results compared to individual features.

Table 2. Confusion matrix for assessing the contribution of angle-based features, density- based features, as compared with the proposed method.

\begin{tabular}{|c|c|c|c|c|c|c|}
\hline \multirow{2}{*}{ Classes } & \multicolumn{2}{|c|}{ Angle-based feature only } & \multicolumn{2}{c|}{ Density-based feature only } & \multicolumn{2}{c|}{ Proposed Method } \\
\cline { 2 - 7 } & Ice & Non-ice & Ice & Non-ice & Ice & Non-ice \\
\hline Ice & $91.26 \%$ & $8.74 \%$ & $92.15 \%$ & $7.84 \%$ & $99.03 \%$ & $0.97 \%$ \\
\hline Non-ice & $6.86 \%$ & $93.13 \%$ & $7.00 \%$ & $93.00 \%$ & $7.92 \%$ & $92.08 \%$ \\
\hline ACR & \multicolumn{2}{|c|}{92.20} & \multicolumn{2}{|c|}{92.57} & \multicolumn{2}{c|}{$\mathbf{9 5 . 5 9}$} \\
\hline
\end{tabular}

\subsection{Experiments on Classification}

Qualitative results of the proposed method for classification are shown in Fig. 15, where we can see successful and unsuccessful classifications of ice and non-ice images. It is observed from Fig. 15 that the proposed method classifies ice and non-ice images correctly, though the images are affected by low contrast, degradations and background variations (indoor and outdoor scenes). However, when ice is covering tree branches and the presence of small amounts of ice covering power transmission lines, the 
proposed method misclassifies some images. This shows that there is scope for further improvement. Quantitative results of the confusion matrix and classification rates of the proposed and existing methods are reported in Table 3, where it is noted that the proposed method is better than the existing method in terms of ACR. The reason for the poor results of the existing method is that the features proposed in that method are good for high contrast images. In addition, since the features such as texture and statistical are generic, when an image does not contain ice regions, the features are not effective for classification. On the other hand, since the proposed method involves robust features, namely, those that are rotation invariant, ice size-independent and contrast-independent, the proposed method is better than the existing method. Besides, the combination of angle and density information makes a difference compared to the existing method.

To show the proposed method is invariant to rotation, some sample images of different rotations are shown in Fig. 16, we also rotate the images in our dataset randomly for computing the confusion matrix and ACR as reported in Table 3. Table 3 shows that the results on the images of different rotations and our dataset are the same. This indicates that the proposed features are invariant to rotation as these features are extracted based on parallel lines to the major and minor axes of candidate components.
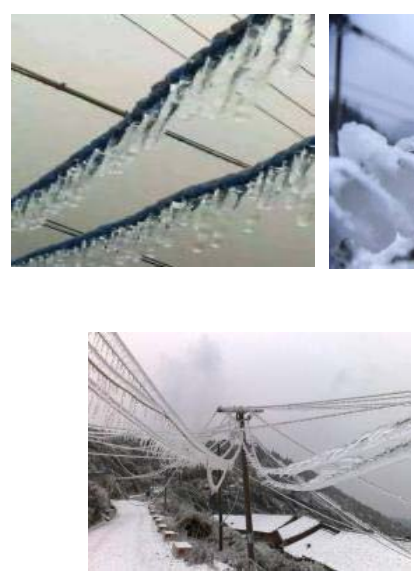

(a) Successful classification based on the proposed method

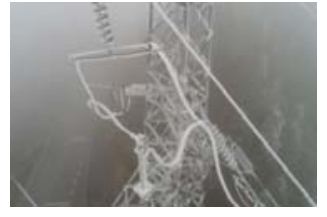

Ice images as non-ice
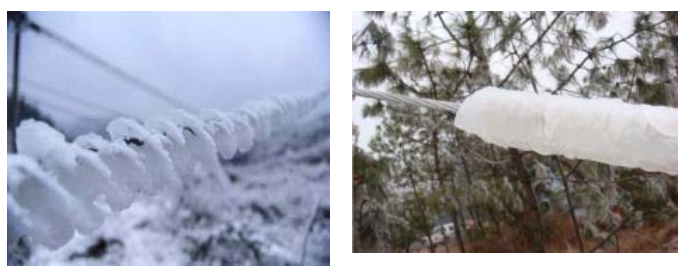

Sample ice images

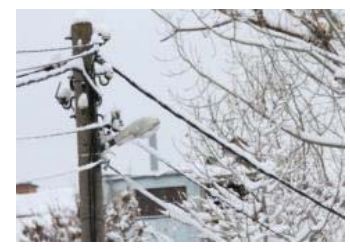

Sample non-ice images
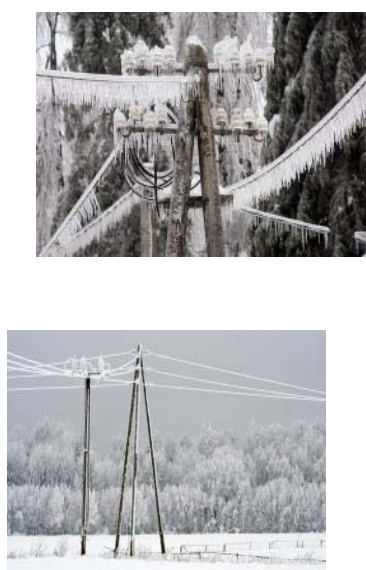
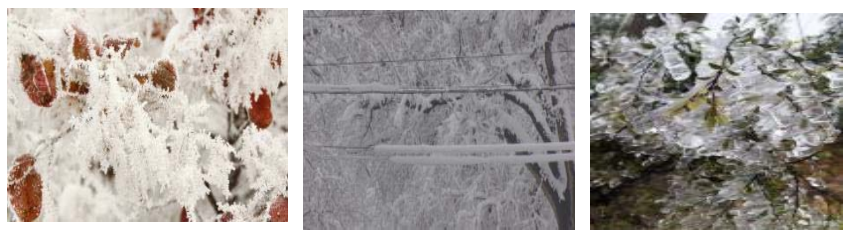

Non-ice images misclassified as ice images.

(b) Unsuccessful classification of the proposed method

Fig. 15. Qualitative results of the proposed classification method.

Table 3. Effectiveness of the proposed method on different experiments (in \%).

\begin{tabular}{|c|c|c|c|c|c|c|}
\hline \multirow{2}{*}{ Classes } & \multicolumn{2}{|c|}{ Proposed method } & \multicolumn{2}{|c|}{ Existing method (Li et al. 2015) } & \multicolumn{2}{|c|}{ Proposed method used for different rotations } \\
\cline { 2 - 7 } & Ice & Non-ice & Ice & Non-ice & Ice & Non-ice \\
\hline Ice & $99.03 \%$ & $0.97 \%$ & $82.52 \%$ & $17.48 \%$ & $99.03 \%$ & $0.97 \%$ \\
\hline
\end{tabular}




\begin{tabular}{|c|c|c|c|c|c|c|}
\hline Non-ice & $7.92 \%$ & $92.08 \%$ & $43.56 \%$ & $56.44 \%$ & $7.92 \%$ & $92.08 \%$ \\
\hline ACR & \multicolumn{2}{|c|}{95.59} & \multicolumn{2}{|c|}{69.46} & \multicolumn{2}{c|}{95.59} \\
\hline
\end{tabular}
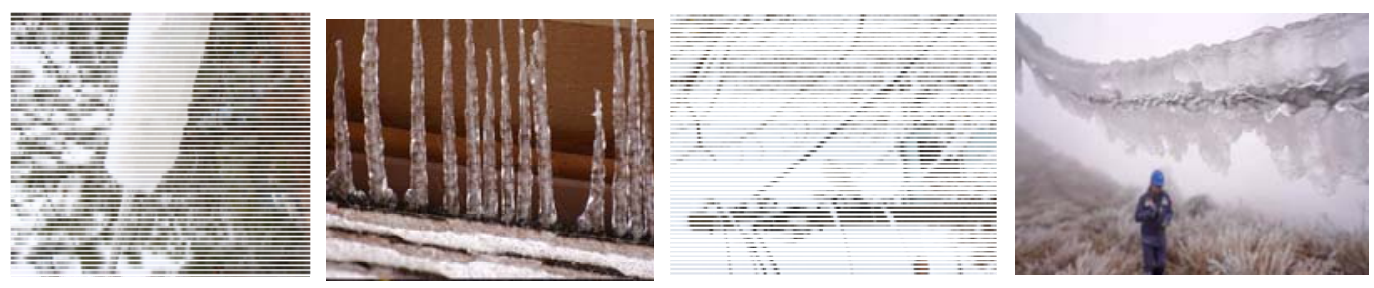

Fig. 16. Sample successful classifications using the proposed method for different rotations

\subsection{Validating the Proposed Classification}

As discussed in the Experimental Section, to demonstrate the advantage of the proposed classification scheme, we run the ice detection method (Jiao, 2010) before and after classification to calculate measures. Sample qualitative results of the ice detection method before and after classification are shown in Fig. 17, where the ice detection results are better for after-classification compared to beforeclassification. This is understandable because when the classification method removes images that have no ice regions from the list of input images, the results of the ice detection method improves automatically due to the lower number of false positives. Further, when the classification method identifies the classes as ice and non-ice, one can modify or tune the same method according to the complexity of classes for achieving better results. In other words, we can choose an appropriate method according to the nature of the problem. In this experiment, we tune the structural element of the morphological operation and the parameters of an SVM classifier of the ice detection method after classification. It is evident from the results reported in Table 4 that the precision and F-score of the ice detection method improves the after-classification compared to before-classification. Further, we note from Table 4 that there is a significant improvement in precision for after-classification as compared to the before-classification results, while recall is the same for both before- and after-classification. This shows that the proposed classification method helps in reducing the number of false positives for the ice detection method. Therefore, we can conclude that the proposed classification method is useful for achieving accurate and better results for ice detection, ice thickness identification and monitoring. 

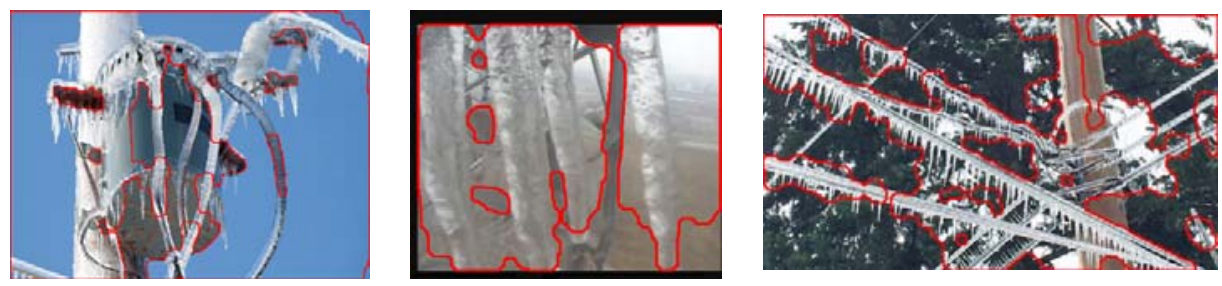

(a) Ice detection by the method in (Jiao et al. 2010) before classification
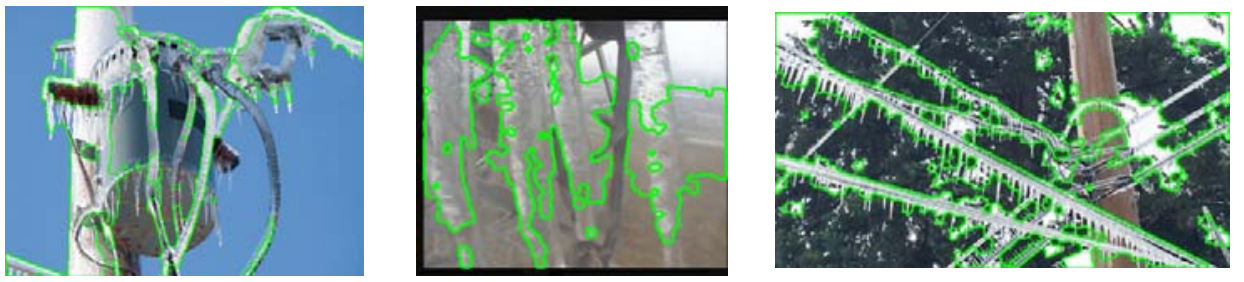

(b) Ice detection by the method (Jiao et al, 2010) after classification

Fig. 17. Performance of ice detection before and after classification

Table 4. Performance of the ice detection method before and after classification.

\begin{tabular}{|c|c|c|c|c|c|c|}
\hline \multirow{2}{*}{ Ice Detection Method } & \multicolumn{3}{|c|}{ Before Classification } & \multicolumn{3}{c|}{ After classification } \\
\cline { 2 - 7 } & Recall & Precision & F-score & Recall & Precision & F-score \\
\hline Jiao et al, 2010 & 0.62 & 0.60 & 0.61 & 0.62 & 0.74 & 0.67 \\
\hline
\end{tabular}

\section{Conclusions and Future Work}

In this work, we have proposed a new expert system for the classification of ice and non-ice images to improve the performance of ice detection, thickness identification and ice thickness monitoring methods. For each input image, the proposed method explores Haar wavelet decomposition for enhancing the finer details in the image by computing the average across three high-frequency sub-bands. This work considers four decomposition levels based on an experimental study, which results in four average images for each input image. For enhanced images, the proposed method obtains Canny edge images to study component shapes in the images. The proposed method performs a morphological operation over Canny edge images to fill a single pixel gap if any, which results in Candidate Component Detection (CCD). For each component in the CCD images, the proposed method finds the major and minor axes, and then extracts angle- and density-based features for pixels that fall on parallel lines to the major and minor axes. The proposed method selects features from each of the wavelet decomposition levels and computes the mean of those features, which results in a feature matrix. Furthermore, the feature matrix is fed to a Multi-Layer Perceptron Neural Network for the classification of ice and non-ice images. Experimental results on classification, and comparative studies with the existing method, show that the proposed method outperforms the latter in terms of classification rate. Similarly, experimental results for ice detection show that the proposed classification is useful for substantially enhancing ice detection performance.

As discussed in the Experimental Results Section, when images are affected by low contrast and little ice information, the proposed method does not perform well. At the same time, when non-ice images contain ice covering other objects such as tree branches, the proposed classification method 
misclassifies the images as an ice one. To overcome the above limitations, we have planned to explore the combination of multiple deep learning models with different feature extraction approaches. In addition, the developed system will be modified such that it can be used for real-time applications. In order to support reproducible research, we plan to release the dataset along with the code after receiving positive feedback from the journal.

\section{Acknowledgement}

The work described in this paper was supported by the Natural Science Foundation of China under Grant No. 61672273 and No. 61272218, the Science Foundation for Distinguished Young Scholars of Jiangsu under Grant No. BK20160021, and the Scientific Foundation of the State Grid Corporation of China (Research on Ice-wind Disaster Feature Recognition and Prediction by Few-shot Machine Learning in Transmission Lines).

\section{References}

Chang, Y., Yu, H. and Kong, L. (2018), Study on the calculation method of ice thickness calculation and wire extraction based on infrared image. In Proc. ICMA, pp 381-386.

Jiao, R., Li, B. and Li, Y. (2010), Detection of cladding ice on transmission line based on SVM and mathematical morphology. In Proc. CSIP, pp 1624-1628.

Li, B., Thomas, G. and Williams, D. (2015), Ice Detection on Electrical Power Cables. Advances in Visual Computing. Springer, pp 355-364.

Li, J., Shao, Q., Xue, K., Wang, C and Hu, W. (2017), The icing-thickness detection of high-voltage transmission line based on machine vision, In Proc. ICIA, pp 381-385.

Liang, G., Shivakumara, P., Lu, T. and Tan, C. L. (2015), A new wavelet-Laplacian decomposition for arbitrarilyoriented character segmentation in video text lines, In Proc. ICDAR, pp 926-930.

Liu, Y., Tang, Z. and Xu, Y. (2017), Detection of ice thickness of high voltage transmission lines by image processing. In Proc. IAEAC, pp 2191-2194.

Nanni, L., Chidoni, S and Brahnam, S., (2018), Ensemble of convolutional neural networks for bioimage classification, Applied Computing and Informatics.

Raghu, S. and Sriraam, N. (2017), Optimal configuration of multilayer perceptron neural network classifier for recognition of intracranial epileptic seizures, Expert Systems with Applications, pp 205-221.

Romeo, J., Pajares, G., Montalvo, M., Guerrero, J. M., Guijarro, M and Cruz, J. M. D. (2013), A new expert system for greenness identification in agricultural images, Expert Systems with Applications, pp 2275-2286.

Sun, G. (2018), Image processing and recognition technology of transmission line icing research. Chemical Engineering Transactions, pp 871-876.

Wang, J., Wang, J., Shao, J. and Li, J. (2017), Image recognition of icing thickness on power transmission lines based on a least squares and Hough transform. Energies.

Yang, M., Cai, L., Feng, J. and Yao, X. (2017), Image recognition of icing thickness on power transmission line based on fractal edge detection. Revista de la faculatad de ingenieria, U. C. V. pp 320-329.

Zeng, X. J., Luo, X. L., Lu, J. Z., Xiong, T. T. and Pan, H. (2012), A novel thickness detection method of ice 
covering on overhead transmission line, Energy procedia, pp 1349-1354.

Zhang, X and Zhu, X. (2019), Autonomous path tracking control of intelligent electric vehicles based on lane detection and optimal preview method, Expert Systems with Applications, pp 38-48.

Zhao, J., Zhao, J., Yan, B and Chen, B. (2018), Icing monitoring system of transmission lines based on image and stress. In Proc. EI2, pp 1-4.

Zhao, Z., Xu, S., Kang, B. H., Kabir, M. M. J. and Liu, Y. (2015), Investigation and improvement of multi-layer perceptron neural networks for credit scoring. Expert Systems with Applications, pp 3508-3516.

Zhong, Y. P., Zuo, Q and Zhang, C. (2013), A new image-based algorithm for icing detection and icing thickness estimation for transmission lines, In Proc, ICMEW, pp 1-6. 